\title{
Hippocampal Sulcus
}

National Cancer Institute

\section{Source}

National Cancer Institute. Hippocampal Sulcus. NCI Thesaurus. Code C32744.

A shallow groove dividing the dentate gyrus from the hippocampal CA1 field and the subiculum. 